\title{
eJRIEPS
}

Ejournal de la recherche sur l'intervention en éducation physique et sport

$34 \mid 2015$

Varia

\section{L'activité de l'enseignant d'EPS entre préoccupations et « occupations » : un point de vue développemental}

Frédéric Saujat et Guillaume Serres

\section{(2) OpenEdition}

Édition électronique

URL : http://journals.openedition.org/ejrieps/1702

DOI : 10.4000/ejrieps.1702

ISSN : 2105-0821

Éditeur

ELLIADD

Référence électronique

Frédéric Saujat et Guillaume Serres, « L'activité de l'enseignant d'EPS entre préoccupations et " occupations » : un point de vue développemental », eJRIEPS [En ligne], 34 | 2015, mis en ligne le 01 janvier 2015, consulté le 07 octobre 2019. URL : http://journals.openedition.org/ejrieps/1702 ; DOI 10.4000/ejrieps. 1702

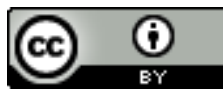

La revue eJRIEPS est mise à disposition selon les termes de la Creative Commons Attribution 4.0 International License. 


\section{eJRIEPS 34 janvier 2015}

\section{L'activité de l'enseignant d'EPS entre préoccupations et "occupations" :}

\section{un point de vue développemental}

Frédéric Saujat* et Guillaume Serres**

* Aix-Marseille Université, EA 4671 ADEF, ENS de Lyon, IFE, Marseille, France

** Clermont Université, EA 4281 ACTé, Clermont-Ferrand, France

\section{Résumé}

Cette contribution soutient que l'activité des enseignants ne saurait être comprise à partir des seules intentionnalités. Elle montre comment les intentions des acteurs sont débordées face aux situations d'enseignement.

L'activité des enseignants d'EPS est appréhendée dans les situations qui supposent de mobiliser et d'entretenir le collectif de et au travail que constituent les élèves. Situations pouvant poser problème à des débutants et à des expérimentés. Des études de cas servent de support à cette contribution. L'activité des enseignants y est envisagée et décrite à deux niveaux selon deux échelles de temps. Elle est envisagée à chaque instant comme un effort déployé pour donner corps à leurs "préoccupations" (ce qu'ils voudraient faire) à travers leurs "occupations " (ce qu'ils font effectivement en situation de travail). Appréhendée dans le temps, au gré de diverses situations, cette activité est également envisagée comme une succession de réorganisations autour d'équilibres provisoires traduisant des formes d'efficacité " objective " et "subjective "différentes.

Les résultats permettent de modéliser le développement de l'activité professionnelle comme une histoire à travers laquelle celle-ci, en se réalisant, laisse derrière elle et devant elle des possibilités non réalisées. Ils permettent de considérer sans les dissocier les intentionnalités et les déterminants à l'œuvre dans l'activité des enseignants. Les implications pour la formation sont envisagées en guise de conclusion.

Mots clés: activité, intentionnalité, préoccupations, occupations, développement de l'activité 


\section{eJRIEPS 34 janvier 2015}

\section{Introduction}

Le texte de cadrage de ce numéro thématique invite à engager une réflexion sur l'activité de l'enseignant d'EPS du point de vue des intentionnalités et des déterminants « qui rendent possible ou au contraire empêchent cette dernière ». Par cette contribution, nous proposons d'interroger cette activité sous l'angle de son développement. Nous souscrivons sans réserve à l'idée que cette activité ne se réduit pas à « une entreprise opératoire ", mais nous en tirons une conséquence : les possibilités et les empêchements de l'activité de l'enseignant d'EPS ne sauraient être compris et/ou expliqués à partir des seules intentionnalités. Ces dernières sont débordées par les épreuves subjectives à la source des compromis au moyen desquels l'enseignant s'efforce de donner corps à ses «préoccupations» à travers ses « occupations». En effet si «la subjectivité, c'est alors uniquement l'insatisfaction. Mais c'est peut-être là la vie elle-même » (Canguilhem, 1983, p. 364), cette « insatisfaction suppose mémoire, frustration, tension vitale, valeurs et évaluation. On comprend alors pourquoi elle peut «déplacer 》 les perspectives» (Schwartz, 1992, p. 252). Cette tension vitale joue un rôle moteur (développemental) dès lors que l'enseignant parvient à convertir celle-ci en intentions mentales qui lui permettent d'organiser la pluralité des cadres de signification de ses actes en produisant ces « déplacements » de perspectives.

Le développement est abordé non pas de façon générale mais en ciblant l'activité orientée vers un but que poursuivent très fréquemment les enseignants, notamment en EPS : « mobiliser et entretenir le collectif de et au travail » que constituent les élèves.

L'analyse de cas d'activité orientée vers la mobilisation et l'entretien de ce collectif de et au travail fait apparaître de multiples leviers de compréhension de son développement. Activité orientée vers la socialisation ou vers l'apprentissage, activité orientée vers les élèves (leurs performances/leurs comportements), vers soi (se rassurer, s'économiser, se ressentir professeur), vers les autres (faire avec les dispositifs et outils du métier pour être reconnu, agir selon des règles de métier dont on ne perçoit pas toujours le sens).

L'étude des réorganisations de ces équilibres provisoires, traduisant des formes d'efficacité «objective » et «subjective » différentes, peut ouvrir alors sur une modélisation d'un développement de l'activité professionnelle conçu comme une histoire à travers laquelle celle-ci en se réalisant laisse derrière elle et devant elle des possibilités subjectives et opératoires non réalisées. 


\section{eJRIEPS 34 janvier 2015}

\section{2. Étudier le développement de l'activité professionnelle : déterminants, équilibres provisoires, intentionnalités, efficacités}

2.1. Les « déterminants » de l'activité enseignante

Le point de vue que nous défendons consiste à affirmer que les principes de l'action des enseignants ne résident pas uniquement dans la situation où se déroule l'interaction, mais qu'il faut les rechercher aussi dans les prescriptions et dans l'activité d'interprétation et de redéfinition à laquelle elles donnent lieu de la part des enseignants. Tout en partageant le point de vue rappelé par Durand (2009) selon lequel « l'activité est irréductible à une commande émanant de prescriptions », nous tenons ces dernières pour consubstantielles du travail des enseignants ; en tant qu'artefacts cognitifs (Mayen \& Savoyant, 2002), elles sont pour nous l'un des organisateurs du processus d'actions et de décisions (Maggi, 2003) à l'œuvre dans le travail enseignant (Saujat, 2011).

De ce point de vue la première tâche consiste à identifier la diversité des sources de prescriptions en jeu dans l'activité professorale. Pour ce faire, nous nous appuierons sur une formalisation (Saujat 2010) mettant en évidence une dynamique articulant quatre niveaux interdépendants :

a) celui de la prescription primaire et secondaire (Goigoux, 2007), et plus largement des « normes antécédentes »(Schwartz, 2000),

b) celui de la réorganisation opérée par les collectifs professionnels qui représente une première mise à l'épreuve de la prescription et débouche sur des obligations ou des règles de métier que se donnent ces collectifs pour faire ce qu'on leur demande de faire,

c) celui de la personnalisation de ces obligations génériques par chaque professionnel qui s'auto-prescrit ce qu'il a à faire,

d) celui du travail réel en classe où cette auto-prescription est mise à l'épreuve de l'activité conjointe de l'enseignant et des élèves.

Si l'on s'appuie sur la distinction proposée par Six (1999) et reprise par Daniellou (2002), on peut caractériser le niveau a) comme celui des prescriptions « descendantes » alors que les niveaux b), c) et d) relèvent de prescriptions « remontantes ».

Les premières sont des prescriptions exogènes et comportent d'une part la prescription primaire, portée par l'organisation hiérarchique du travail enseignant (programmes, instructions et recommandations pédagogiques, évaluation du travail enseignant par les corps d'inspection et évaluation des performances des élèves), d'autre part la prescription secondaire élaborée et diffusée par la formation à travers des reformulations, interprétations ou concrétisations des injonctions hiérarchiques mais aussi à travers la 


\section{eJRIEPS 34 janvier 2015}

production de recommandations autonomes (Daguzon et Goigoux, 2007 ; Goigoux, 2007). En utilisant plus haut la notion de «normes antécédentes » pour désigner ce premier niveau, nous avions en vue avec Schwartz (2000) la nécessité d'étendre le périmètre circonscrit par ces deux types de prescriptions «aux acquis de l'intelligence et de l'expérience collective » (p. 598), aux savoirs «inopératifs » et aux savoirs professionnels de référence qui contribuent à fonder la professionnalité enseignante. II faut souligner ici que les évolutions récentes de ces prescriptions descendantes, et tout particulièrement de la prescription primaire, se caractérisent non seulement par un brouillage croissant des finalités de l'Ecole, dont la responsabilité est de moins en moins « limitée » - transmettre des connaissances, faire construire des compétences, préparer à « l'insertion » et à «l'employabilité », compenser les déficits de « socialisation » dont une fraction croissante de la population scolaire serait porteuse, former à la « citoyenneté »...- mais aussi par un élargissement et une diversification des missions, des fonctions et des tâches assignées à ses professionnels, à travers une nouvelle division sociale du travail entre les différents partenaires éducatifs et une recomposition des milieux de travail enseignant.

On conçoit aisément le type de conflits de critères qu'entraîne une telle prescription, qui tend de plus en plus à se rapprocher d'une « prescription infinie » (Daniellou, 2002) dont les effets sont d'autant plus lourds sur la signification sociale et l'organisation du travail enseignant qu'elle s'inscrit désormais dans une «culture de la performance » et dans une logique d'obligation de résultats aux critères d'évaluation de plus en plus incertains, hétérogènes voire contradictoires. Si l'on ajoute que cette prescription infinie des objectifs va de pair avec une sous-prescription chronique des moyens pour les atteindre, on conviendra qu'il y a là de quoi compliquer la tâche à ces derniers.

Mais les enseignants ne sont pas confrontés seulement à ces prescriptions « descendantes », ils doivent compter également avec des prescriptions « remontantes », correspondant aux niveaux b), c) et d). Ces dernières ne résultent pas de la structure organisationnelle du travail enseignant ou de la formation, mais des collectifs de travail, des enseignants et de l'objet du travail lui-même.

Les prescriptions qui « remontent » du collectif de travail correspondent au niveau b), celui de la production endogène d'obligations génériques. Ce travail d'organisation qu'opèrent les collectifs, première mise à l'épreuve des prescriptions descendantes en vue de les " digérer », est aujourd'hui bien souvent contrarié par ces dernières. La défaillance fréquente de ces prescriptions endogènes renvoie les enseignants à eux-mêmes, les contraignant à trier tout seuls entre les différentes sources de prescription, qu'elles 


\section{eJRIEPS 34 janvier 2015}

viennent « d'en haut » ou « d'en bas ». II leur faut alors établir des priorités sans jamais pouvoir les satisfaire toutes (Daniellou, 2002) et arbitrer à la recherche d'une efficacité "malgré tout", qui questionne le métier, sur les plans éthique et technique, dans sa capacité à dépasser les contradictions qui révèlent ses potentialités et ses lacunes face à des situations professionnelles inédites. Privé des « débats de normes " (Schwartz, 2000) constitutifs de ce niveau, l'enseignant se voit contraint de reporter les « renormalisations " nécessaires en aval, aux niveaux $\mathrm{c}$ ) et d), et de les opérer pour son propre compte en l'absence de répondant collectif.

Les prescriptions "remontant » de l'enseignant lui-même relèvent du niveau c) et sont liées aux auto-prescriptions qu'il se donne. L'élaboration de ces dernières est tissée dans un ensemble de choix -et donc de renoncements- et dans un retravail de ses raisons et moyens d'agir constitutif du rapport subjectif à sa tâche. Ce niveau, relevant du travail de préparation, revêt un statut particulier en ce qu'il occupe une position charnière entre les prescriptions des niveaux a) et b) et le travail réel en présence des élèves. Ce statut résulte en effet d'une caractéristique de l'activité du professeur: soumis à des prescriptions, il lui revient d'en élaborer à son tour à l'intention de ses élèves. Du coup, lorsque l'enseignant prépare sa classe et s'auto-prescrit la tâche qui organisera son travail en présence des élèves, il se livre bien à un travail réel qui représente une deuxième mise à l'épreuve des prescriptions descendantes, après celle que leur a fait subir le niveau b). Mais une fois ce travail de préparation réalisé, celui-ci devient à nouveau du prescrit pour l'enseignant. II s'agit alors d'un prescrit adressé à lui-même (ce qu'il se demande) mais également à ses élèves (ce qu'il leur demande).

Les prescriptions "remontant» de l'objet du travail, qui a connu des transformations sensibles ces dernières années, correspondent au niveau d). Elles sont relatives à la « matière " humaine avec, pour et sur laquelle travaillent les enseignants. II s'agit de ce à quoi ils sont occupés lorsqu'ils font classe mais aussi de ce par quoi ils sont occupés : comment organiser les conditions de l'étude avec des élèves dont le rapport à l'expérience scolaire a perdu de son immédiateté, comment gérer l'hétérogénéité, comment faire avec les problèmes de discipline, les élèves « incontrôlables ", les difficultés ou les résistances à apprendre, les échecs d'une partie des élèves, les refus de l'école et les décrochages... ? Le travail réel qui se déroule en classe, travail en co-activité avec les élèves, constitue ainsi une troisième mise à l'épreuve des prescriptions, après celles engendrées respectivement par les niveaux b) et c). Si l'activité déployée au niveau précédent a consisté à concevoir et à organiser au mieux son travail et celui des élèves, 


\section{eJRIEPS 34 janvier 2015}

cette activité de conception et d'organisation se poursuit dans l'activité conjointe avec eux. Le professeur devient alors le gestionnaire et l'opérateur de sa propre prescription, qui connaît donc une nouvelle transformation, en fonction des effets qu'elle produit sur l'activité des élèves mais aussi sur sa propre activité, dans sa façon de "prendre ", de «faire » la classe et de la reconcevoir pour la fois suivante. II incombe au professeur de mettre les élèves au travail, en gérant un processus de "négociations " de la tâche dans le cadre d'une activité collective dont l'objet est la régulation de l'accomplissement de ce travail et de sa mise en perspective. Ces transformations de la prescription, en temps réel dans la relation avec la classe, ou après-coup en vue de la séance suivante, constituent une face cachée du travail enseignant qui est un moyen de régulation des conditions d'enseignement-apprentissage tout aussi important que la régulation de l'activité des élèves pendant la leçon. De ce point de vue les métamorphoses que connaît la tâche prescrite aux élèves au cours de ce processus révèlent une fonctionnalité particulière de cette dernière dans le travail d'enseignement-apprentissage. En effet l'intersubjectivité qui tisse la relation didactique est toujours prise, pour reprendre une expression utilisée par Latour dans un autre cadre, dans une "interobjectivité » liée à la présence d'une tâche, qui supporte les objets et les enjeux de savoir et de formation. L'interaction avec autrui, qui définit selon Tardif et Lessard (1999) l'objet du travail enseignant, est donc toujours médiée par cette tâche, dont l'enseignant comme les élèves cherchent à faire l'instrument de leur activité. La tâche scolaire apparaît ainsi à la fois comme l'objet et l'outil du travail de l'enseignant et des élèves : c'est elle qui permet au premier d'organiser un milieu de travail qui mobilise les seconds dans une réalisation collective. Mais il faut tirer ici toutes les conséquences d'une conception instrumentale de la tâche (Vérillon, 2005). La tâche prescrite aux élèves peut être regardée comme un dispositif (humain, matériel, temporel, spatial, et symbolique) adressé aux élèves et visant à obtenir d'eux un travail scolaire. A ce titre elle constitue " un instrument "bi-face" à la disposition d'acteurs engagés dans une collaboration asymétrique mais à visée partagée [...] et à travers lequel chacun devrait trouver un point d'appui pour développer son pouvoir d'agir sur la situation » (Vérillon, 2005, p. 4).

L'activité de l'enseignant ne saurait donc être regardée uniquement comme une réponse aux prescriptions, qui à l'opposé de normes externes auxquelles se confronterait le professeur, sont consubstantielles de son activité (Amigues, Félix et Saujat, 2008). II s'agit bien plutôt d'un dialogue avec les activités de conception et de re-conception à la source 


\section{eJRIEPS 34 janvier 2015}

de ces dernières, dialogue qui se poursuit comme on vient de le voir jusque dans l'interaction avec les élèves.

\subsection{L'enseignement comme travail}

Le travail de l'enseignant consiste donc, à partir des prescriptions exogènes (primaire et secondaire) et endogènes, à prescrire des tâches visant des transformations des élèves sur les plans moteur, affectif, cognitif, identitaire et social. Ce travail peut être défini comme la gestion d'une mise en activité collective des élèves, depuis la conception de cette activité collective, qui doit permettre un apprentissage et un développement personnels de chacun, jusqu'à sa réalisation en situation. Mais justement cette réalisation n'est jamais totalement prédictible. Durand (1999) attire notre attention sur le fait que «pour qui s'intéresse à l'analyse du travail, l'enseignement s'apparente à celui de l'ergonome. En effet, une part de cette activité consiste à définir, organiser, réguler un travail : celui des élèves. Les enseignants sont des pourvoyeurs de tâches et les modélisations de l'apprentissage scolaire et de l'instruction, mettent l'accent sur cette notion de travail, opérationnalisée en termes d'engagement dans une tâche » (p. 124). Ce qui précède alimente bien ce point de vue sur le travail de l'enseignant. Néanmoins, on peut être plus précis encore dans le choix des activités de l'ergonome susceptibles de soutenir une telle comparaison, en observant que, toutes proportions gardées, le professeur se trouve devant une difficulté comparable à celle de l'ergonome de conception qui tente d'évaluer et d'anticiper « l'espace des formes possibles de l'activité future » (Daniellou, 1992), celle de ses élèves mais aussi la sienne propre en réponse à la leur. La prévision de cette activité future constitue un défi toujours renouvelé, dès lors que les tâches proposées ne sont que des prescriptions d'intentions et de possibilités d'actions qu'il revient aux sujets agissants de s'approprier et d'opérationnaliser. La prévision dépend du sens, imprédictible, que les élèves attribueront à la tâche prescrite par l'enseignant. Pour autant, l'appropriation de la tâche par les élèves ne saurait être laissée au hasard, il incombe au professeur de l'organiser en construisant un « espace des formes possibles » de son activité conjointe avec les élèves : un milieu qui fixe des contraintes et offre des ressources susceptibles d'être saisies comme des occasions de développement de cette activité conjointe (Amigues, Faïta et Saujat, 2004).

L'enseignant est donc placé dans la situation paradoxale d'un prescripteur de tâches, d'un organisateur du travail qui serait contraint, en temps réel, de réorganiser ces tâches en fonction de l'activité des élèves. Paradoxale aussi parce que l'enseignant devrait simultanément initier, maintenir et développer une dynamique collective autour de la 


\section{eJRIEPS 34 janvier 2015}

réalisation de ces tâches, lors même qu'elles connaissent des transformations plus ou moins profondes dans la situation d'interaction avec les élèves en fonction de leurs manières différenciées de faire (ou de ne pas faire) ce qui leur est prescrit. L'enseignant est ainsi le sujet d'une double activité, en ce que sa soumission à la prescription le contraint à organiser l'activité des élèves suivant des schémas, et avec des moyens dont le respect et l'usage sont autant d'épreuves pour les élèves que pour lui qui est censé anticiper sur le déroulement du processus engagé. II doit définir le travail d'autrui tout en définissant les moyens qu'il va se donner de conduire ce travail à son terme. Cette caractéristique fait de lui un prescripteur-opérateur.

En conséquence, si l'organisation de l'apprentissage constitue bien l'objet du travail du professeur, on n'aura garde de sous-estimer ce que ça lui demande du point de vue de son activité de viser un tel "horizon», qui à l'instar de la guérison du patient pour le médecin, représente pour lui un but lointain et incertain, vers lequel tend son action et qui implique la coopération des élèves, là encore à l'instar de la guérison qui ne peut advenir sans celle du patient. D'une part parce que cet "horizon" ne pourra jamais être réellement atteint : Tardif et Lessard (1999, p. 226) notent fort justement que la tâche d'enseignement comporte « une forte composante dynamique et historique car elle remplit moins un programme d'actions tout défini d'avance qu'elle rend possible, en quelque sorte, le futur en le réalisant ». D'autre part parce que "réaliser le futur » nécessite un investissement qui a un coût pour l'enseignant, dans la mesure où il est contraint d'agir en fonction de situations qu'il doit créer mais dont les objectifs ne sont ni immédiats ni précis, et donc de se fonder sur des anticipations qui dépendent des initiatives et des décisions qu'il prend dans le présent, sans jamais être assuré de leur pertinence actuelle et a fortiori future, d'autant plus que ce "bricolage interprétatif et stratégique [...] sous-tend aussi l'activité des élèves et leur engagement dans l'interaction scolaire » (Nonnon, 2000, p. 85).

2.3. Conflictualité, production de ressources et dynamique de l'intentionnalité dans l'activité enseignante

On peut alors comprendre le "sentiment de fatigue " qui résulte souvent d'un exercice professionnel soumis à une telle " charge psychique », en particulier dans les «zones de turbulences " où se trouvent bien souvent propulsés les enseignants débutants (Saujat, Faïta et Maggi, 2006). Le sentiment de division à l'intérieur d'eux-mêmes qu'ils éprouvent fréquemment est lié aux conflits entre ce qu'ils voudraient faire et l'usage qu'ils font d'euxmêmes pour faire ce qui est à faire. L'exposition à des difficultés similaires, le fait de rencontrer des obstacles comparables dans la mise en œuvre des gestes professionnels 


\section{eJRIEPS 34 janvier 2015}

de conduite de la classe, génèrent alors des stratégies qui, malgré la dispersion géographique liée aux lieux d'exercice de ces jeunes professeurs et les spécificités liées à leurs disciplines d'enseignement (Ria, 2004 ; Roustan et Saujat, 2008 ; Saujat, 2004, 2008, 2011), présentent des traits communs. Ils cherchent à « surcompenser » (Vygotski, 1994) leur difficulté provisoire à gérer des situations complexes par le développement de ressources intermédiaires au moyen desquelles ils s'efforcent d'instaurer un cadre qui rende possible non seulement l'apprentissage des élèves, mais aussi leur propre apprentissage du métier, au prix de renoncer, fût-ce provisoirement, à ce qu'ils voudraient faire.

Ces ressources intermédiaires sont élaborées pour surmonter les difficultés inhérentes à la non maîtrise provisoire des genres caractérisant le travail des enseignants expérimentés et à l'impossibilité consécutive de mobiliser efficacement ces composantes génériques, provisoirement hors de leur zone de développement professionnel potentiel. Par exemple les enseignants expérimentés commencent par « prendre » leur classe (en ritualisant fortement l'entrée en classe : déplacements, installation des élèves à leur poste de travail, appel plus ou moins solennel, rappel du cours précédent, sollicitations verbales des élèves, etc.) pour pouvoir « faire » classe (mettre les élèves au travail et les engager dans l'apprentissage). II est au contraire fréquent de voir les enseignants débutants, confrontés à des difficultés récurrentes de mise en œuvre des rituels permettant de «prendre » la classe, inverser l'ordre des composantes génériques constitutives de l'activité de ceux qui sont du métier. Ils commencent ainsi par «faire » la classe pour pouvoir la «prendre » : ils mettent rapidement les élèves au travail en vue d'enrôler ces derniers dans des tâches, à partir de consignes minimales. Mais du coup cette mise au travail n'a pas la même fonction que chez les enseignants expérimentés, pour qui elle est tournée vers l'apprentissage des élèves, alors que dans le cas des débutants elle constitue un moyen de régler des problèmes de prise en main de la classe, sources de préoccupations vives pour eux. Ces ressources intermédiaires sont donc constituées de manières d'agir et de penser qui, pour se nourrir des « genres de l'activité professorale » à travers le dialogue avec leurs pairs chevronnés, n'en sont pas moins propres aux enseignants débutants en tant que produits de leur activité et instruments de leur pouvoir d'agir. Les enseignants débutants ne se contentent pas de vivre dans le contexte, souvent anxiogène, où ils exercent et apprennent à la fois leur métier (Ria, 2009a ; Roustan et Saujat, 2008 ; Saujat, 2004). Ils produisent du contexte pour vivre (Clot, 2008), et cette production de contexte est «marquée » collectivement. Non pas au sens où elle porterait 


\section{eJRIEPS 34 janvier 2015}

la marque d'un collectif de débutants, mais au sens où les contextes produits sont porteurs de caractéristiques qui attestent leurs préoccupations communes de se protéger des "agressions" de leur milieu de travail, d'anticiper leurs difficultés à anticiper et de contenir les effets déstabilisants des inattendus auxquels ne cesse de les confronter ce milieu, en multipliant les attendus.

Cela contribue à donner une tournure spécifique à leurs façons de faire qui les constitue en une communauté proche du type de celle, linguistique, distinguée par Bakhtine (1984). II faut donc voir dans ce détournement de ressources des manifestations de leur intentionnalité, de leur vouloir-faire - Bakhtine parle de son côté de vouloir-dire ou de dessein discursif - matérialisées dans une somme d'éléments épars qui finissent par entretenir entre eux des relations génériques caractérisant des configurations d'activité provisoirement stabilisées, dans lesquelles se reconnaissent les enseignants débutants. Cette activité commune à un sous-groupe professionnel - la catégorie " débutants " au sein du corps enseignant -, constituée autour de préoccupations particulières relatives à l'entrée dans les métiers de l'enseignement, autorise alors à parler de "genre débutants ", qu'il faut entendre plus exactement comme un usage débutant du genre professionnel enseignant.

Cette hypothèse d'un usage débutant du genre ne doit pas conduire à penser que ce "genre débutants " serait constitué de façons de faire normalisées dont on pourrait dresser l'inventaire, ni même qu'il serait attaché à l'entrée dans le métier et qu'il faudrait par conséquent concevoir autant de genres ou d'usages du genre que de phases dans le développement professionnel, ce qu'infirme le fait que le " genre débutants » ne concerne pas que les débutants, fait paradoxal en apparence seulement sur lequel nous aurons l'occasion de revenir ; mais que les ressources intermédiaires qui le caractérisent résultent du dévoiement de ressources expertes qui prennent par destination, au sens juridique du terme, des fonctions différentes au sein de leur activité. Ce dévoiement de ressources professionnelles expertes témoigne donc de la capacité de sujets compétents et créatifs (Rubinstein, 2007), même s'il s'agit d'une compétence à l'état naissant, à agir sur les composantes du genre professionnel enseignant pour les refonctionnaliser. $\mathrm{Ce}$ dévoiement atteste également des attentes communes de ces jeunes enseignants à l'égard de ce même genre professionnel, et par voie de conséquence du fait que ce dernier n'est pas seulement à l'œuvre à travers des exigences qu'il ferait peser sur eux. Autrement dit le vouloir-faire de l'enseignant débutant détermine un usage du genre dans lequel il va pouvoir se réaliser. Ce faisant il dessine des ensembles ouverts de conduites 


\section{eJRIEPS 34 janvier 2015}

et de façons de faire, qui vont entrer en résonance chez les débutants parce qu'elles achèvent, au sens bakhtinien du terme, une totalité qui fonde cet usage (Roustan et Saujat, 2008 ; Saujat, 2004, 2011).

Nous avons pu constater à maintes reprises à quel point ces conduites, les intentions matérialisées qu'elles révèlent, faisaient sens pour ces jeunes enseignants, bien au-delà de l'activité de ceux qui les mettaient en œuvre et dont témoignaient les films servant de support aux interventions ou aux formations auprès d'eux. Nous avons pu vérifier également la productivité de ces manières partagées d'éprouver, de sentir, de penser et d'agir comme «recours pour éviter d'errer tout seul [...] devant l'étendue infinie des bêtises possibles " (Darré, 1994, p.22). II nous semble donc fondé de considérer ce " genre débutants » comme un artefact à caractère social, c'est-à-dire existant en dehors des choix que peuvent en faire les sujets agissants et préexistant à ceux-ci, mais prenant corps par l'insertion dans leur activité. Un artefact qui ce faisant devient un instrument ouvrant à la fois sur la possibilité d'un nouveau rapport d'intelligibilité à leur propre expérience de travail en leur permettant de la resignifier, et sur le développement de leur pouvoir d'agir. Le " genre débutant » correspond ainsi à un moment dans le processus de développement de l'activité, résultant de la nécessité d'agir sur soi à laquelle l'enseignant débutant se trouve confronté. Le "genre débutants " apparaît bien ainsi, à la manière du genre de discours chez Bakhtine, comme une forme de répétition de l'activité enseignante en transformation, comme un «invariant provisoire " reposant sur des équilibres non moins provisoires et vectorisé par le développement de cette dernière (Roustan et Saujat, 2008 ; Saujat, 2010, 2011). Ce qui, au moment d'entrer dans le métier, constitue pour les débutants un objet central de préoccupations et d'attention partagées, peut se trouver relégué sous l'effet de l'activité par laquelle ils s'efforcent d'avoir du métier, au rang de routines «modularisées " et intégrées à des actions plus complexes (Bruner, 1983), constitutives du répertoire de ceux qui sont du métier.

Ces actions plus complexes mobilisent d'autres opérations au service d'autres buts, convoquant l'activité du professeur à s'inscrire dans un autre "genre ", résultant du «recyclage» de ses préoccupations dans des occupations renouvelées : ce qui avait valeur de principe de l'action peut alors devenir condition d'une action répondant à d'autres significations et réclamant de nouvelles ressources opératoires. C'est ainsi qu'ils forgent les instruments nécessaires pour gérer des situations professionnelles problématiques, pour les faire évoluer, mais aussi pour retirer de ces situations des éléments de leur propre développement. 


\section{eJRIEPS 34 janvier 2015}

Ces instruments résultant d'un usage débutant du genre ont donc une double orientation, centrifuge et centripète (Saujat, 2010, 2011), au service de formes d'efficacité dynamique objective et subjective. En effet si l'on considère qu'il y a dans l'activité enseignante une activité relative aux élèves et une activité relative à soi-même, la caractéristique commune à ces ressources intermédiaires réside dans le fait qu'elles fonctionnent comme des instruments psychologiques tournés au premier degré vers l'activité relative à soi-même et au second degré seulement vers l'activité relative aux élèves. Ce n'est que lorsqu'ils auront atteint un premier palier d'efficacité conditionné par un usage de soi générant confort et plaisir professionnels, que les élèves deviendront l'objet de leur activité et qu'ils s'interrogeront sur les modalités selon lesquelles ils peuvent eux-mêmes constituer des moyens plus efficaces au service de cette activité. II en va de même pour le statut de la tâche prescrite aux élèves dans leur activité. Cette dernière est d'abord pour eux un moyen de prendre la classe en fournissant aux élèves un objet qui finalise et organise leur travail, avant d'être un objet de conception didactique qui devienne un moyen d'apprentissage pour les élèves (Roustan et Saujat, 2008).

Le développement de l'activité des enseignants débutants se fraie donc un chemin à travers des conflits vitaux, dont ils s'efforcent de se déprendre en les renversant en intentions mentales (Clot, 2011 ; Saujat, 2011), qu'il leur faudra rendre opérationnelles à la faveur d'une "subjectivité agissante qui, en même temps, ne tourne jamais le dos à l'efficacité de l'action dans le monde » (Clot, 1999/2002, p. 207).

De ce point de vue, être efficace ce n'est pas seulement atteindre les buts fixés, mais c'est tout autant produire de nouveaux buts dans le cours de l'action et, pour éviter que ces derniers ne restent des "vues de l'esprit », créer les conditions d'efficience nécessaires à leur réalisation voire à leur transformation. Ainsi conçue l'efficacité contribue au renouvellement des fins et des moyens (Clot, 2011).

\section{3. Études de cas}

Nous présentons plusieurs études de cas réalisées auprès d'enseignants en EPS. Certaines ont fait l'objet de travaux antérieurs (Laurent et Saujat, à paraître ; Serres, Ria et Adé, 2004). Trois concernent Caroline (débutante en toute fin de formation) et une Jérôme (dix ans d'expérience). Si elles ont été produites selon des méthodologies et des conditions différentes elles ont en commun de décrire l'activité pas à pas en mettant en évidence, à l'échelle de plusieurs semaines, des réorganisations d'équilibres provisoires objet présenté précédemment - que nous cherchons à caractériser. Le croisement de ces 


\section{eJRIEPS 34 janvier 2015}

études de cas dans le cadre de cet article permet également de mettre en comparaison l'activité d'une novice et d'un expérimenté ce qui permet de voir que leurs situations se ressemblent sous certaines conditions.

Les méthodes mobilisées en amont de la construction des cas sont indirectes. Pour chacun des enseignants, nous avons recueilli des traces de leur activité (supports papiers, enregistrements vidéos) lors de divers temps : leçons, entretien de formation, préparation de leçon, entretien réflexif, etc. A la suite de ces enregistrements, les acteurs ont été confrontés aux traces de leurs actions passées. Nous présentons de façon synthétique ces cas de façon chronologique.

\section{CAS n ${ }^{\circ} 1$ - Caroline :}

Depuis le début de l'année scolaire, Caroline installe le matériel de gymnastique seule avant la leçon. Elle estime ne pas pouvoir déléguer aux élèves cette tâche sans s'exposer à des comportements d'indiscipline. Elle reconnaît cependant ne pas être satisfaite de sa façon de procéder en évoquant la difficulté et la durée de cette installation avant et après chaque cours.

Lors d'un entretien post-leçon, son formateur l'interpelle: «Donc tu installes tout le matériel avant la leçon, avant l'arrivée des élèves ? ». Le professeur stagiaire répond : «Mmm... Oui, je n'ai pas réussi à leur apprendre à installer seuls le matériel, c'est quelque chose que j'appréhende, les élèves sont dissipés et le matériel lourd peut être dangereux... ». La remarque du formateur lui procure un sentiment de malaise : " Je suis mal à l'aise parce que je sens bien qu'il me dit : "C'est pas normal [de procéder de la sorte]... ". En plus au fond de moi je sais que c'est pas comme ça que je vais leur apprendre à être autonomes ». Le formateur lui fait une proposition : "Tu pourrais, sans tout leur faire installer, leur déléguer l'installation d'une partie du matériel... ». Le professeur stagiaire accueille favorablement cette proposition : "C'est pas mal, ça peut être une première étape ».

Elle s'interroge ensuite à plusieurs reprises : “L'histoire du matériel, j'y ai repensé en sortant de l'entretien, puis les jours avant de préparer ma leçon et le jour où j'ai rédigé sur ma feuille mon plan de leçon ». Elle envisage au moment de la rédaction de son plan de leçon d'exploiter la connaissance qu'ont les élèves de la disposition du matériel, et constitue des couples d'élèves complémentaires : un élève "calme » et un "excité ", ou un élève "autonome » et un élève "ayant besoin d'être guidé ». Cette répartition des 


\section{eJRIEPS 34 janvier 2015}

élèves est pour elle une façon de déléguer l'installation d'une partie du matériel (les tapis légers et peu dangereux) et de maintenir le contrôle de la classe.

Au cours de la leçon suivante, le professeur stagiaire, munie du plan de leçon rédigé lors de la préparation, s'adresse aux élèves. Elle indique le rôle des élèves répartis en couples qui, à mesure qu'ils sont nommés, se dirigent vers le matériel qu'ils mettent en place sans incident: "Là, j'envoie les élèves chercher le matériel... Je ne suis pas à l'aise, j'appréhende, même si j'ai tout préparé... ». Une fois les rôles distribués, elle intervient ponctuellement à distance tout en restant à proximité des élèves non impliqués dans cette installation. Finalement, cette nouvelle façon d'intervenir s'accompagne d'un sentiment de satisfaction : "Là, une fois que le matériel est en place, je me dis que c'est pas si mal, c'est même bien ".

Depuis le début de l'année, elle était face à des préoccupations contradictoires : conserver le contrôle et assurer la sécurité de la classe en ne l'impliquant pas dans l'installation du matériel en contrepartie d'une fatigue importante pour elle, ou l'impliquer en prenant des risques au niveau de la sécurité des élèves. Elle a privilégié la première option, mais aucune des deux n'était satisfaisante à ses yeux. Le formateur a mis au jour l'inadéquation de cette activité et lui a proposé une solution. Le professeur stagiaire a retenu cette dernière et y a réfléchi à plusieurs reprises. Elle a ainsi précisé son activité au cours de la préparation de leçon et a concrétisé cette réflexion par l'inscription de couples d'élèves complémentaires sur son plan de leçon. Cette activité a fait intervenir des connaissances issues de ses expériences en situation d'enseignement: connaissances relatives aux caractéristiques des élèves ; connaissances relatives au fait que les élèves présents lors de la leçon précédente ont des repères sur l'emplacement du matériel. C'est dans cet entre-deux, hors des contextes de formation et de classe, que le professeur stagiaire a créé les conditions favorables à cette délégation du matériel. La sélection d'un groupe d'élèves et leur répartition en couples complémentaires ont réduit, pour elle, les risques de perte de contrôle en classe lors de cette installation du matériel.

Les réorganisations successives s'accompagnent d'une fluctuation émotionnelle allant de l'insatisfaction provoquée par le formateur à l'inquiétude de devoir changer son intervention jusqu'à la satisfaction liée à son efficacité en classe. Ce passage d'un équilibre provisoire à un autre est abordé par les professeurs stagiaires avec une grande prudence. Les transformations de l'intervention en classe génèrent de l'incertitude et prennent la forme de "retouches " successives apportées à des procédures testées par le passé. Ces moments de transitions entre deux équilibres s'accompagnent d'une 


\section{eJRIEPS 34 janvier 2015}

nouvelle partition entre efficacité objective et subjective. Soulignons notamment que l'efficacité subjective et objective est augmentée en lien avec l'atteinte de buts jusqu'alors non réalisés.

\section{CAS n०2 - Caroline :}

Depuis le début de son cycle de gymnastique, Caroline s'énerve lorsqu'elle souhaite regrouper les élèves afin qu'ils réalisent des étirements assis chacun sur un tapis de gymnastique : «Là, je suis énervée et je me dis qu'ils ne sont pas autonomes, à chaque fois ils se dispersent dans la salle ». Décrivant le caractère récurrent de cette situation et l'énervement qui lui est lié, elle estime les élèves incapables de se répartir seuls tout en restant suffisamment groupés pour écouter ses consignes.

$\mathrm{Au}$ cours de l'entretien de formation post-leçon, Caroline affirme ne pas trouver de solution: « Je ne vois pas comment faire pour les regrouper ». Le formateur lui suggère : «Pourquoi ne pas utiliser les lignes au sol comme repères ? ». Elle réfléchit alors à haute voix en pointant du doigt les lignes du gymnase sur l'écran de télévision : « ...Oui, quatre tapis entre ces deux lignes, quatre autres tapis ici... ». Lors de l'entretien d'autoconfrontation, elle commente sa réaction faisant suite à la proposition du formateur: «Quand il me dit: "Pourquoi ne pas utiliser les lignes au sol ? », je me demande pourquoi je n'y ai pas pensé avant. Je me dis : "Mais bien sûr! C'est évident, c'est un repère concret pour eux, moi je leur disais de ne pas laisser d'espaces libres, mais c'est pas concret. Si je leur dis dans tel carré [défini par les lignes du gymnase], là c'est concret...». Convaincue par la proposition du formateur, elle est surprise et même vexée de ne pas y avoir pensé seule. Elle énonce les raisons de l'inefficacité de sa procédure et invalide la connaissance mobilisée lors de la LE9ON : La sollicitation verbale suffit à regrouper les élèves et construit une nouvelle connaissance avec l'aide du formateur : L'utilisation de repères concrets au sol est un moyen de regrouper les élèves.

En sortant de l'entretien de formation, le professeur stagiaire précise le déroulement de son activité future : «Je me suis dit: «II faudra que j'indique les limites avant de les envoyer chercher les tapis » ». Au cours de la leçon, le professeur stagiaire demande aux élèves d'aller chercher le matériel nécessaire à leurs étirements. Elle s'aperçoit à leur arrivée qu'elle ne leur a pas donné d'indications particulières concernant leur répartition dans l'espace : «Et là, je les vois arriver et je me suis dis : “Mince ! J'avais prévu de leur donner les limites avant de les envoyer chercher les tapis et là... Pouf ! J'ai oublié... Dans l'action, j'ai complètement oublié... ». A cet instant, elle demande aux élèves les plus 


\section{eJRIEPS 34 janvier 2015}

proches d'elle de se positionner entre deux lignes au sol et essaie d'indiquer ces mêmes repères aux autres élèves dispersés dans le gymnase: "J'indique les limites mais les élèves sont déjà dispersés, ils ne m'entendent pas et ce n'est pas de leur faute ».

L'activité du professeur stagiaire se caractérise par une discontinuité entre l'interaction avec le formateur et l'intervention professionnelle. Nous pouvons penser que cet «oubli » est lié ses préoccupations au moment d'envoyer les élèves chercher leurs tapis. En effet, dès que les élèves se dirigent vers le matériel, elle s'adresse à un élève (Gino) en lui demandant de venir se placer dès qu'il a pris son tapis. La connaissance mobilisée dans cette situation est la suivante : Gino profite à chaque leçon de l'installation des tapis pour chahuter et perturber le déroulement du cours. A cet instant sa préoccupation principale concerne la surveillance d'un élève leader, qui profite à chaque leçon de l'installation des tapis pour chahuter. Cette focalisation, en plus de sa préoccupation d'anticiper l'exercice suivant, l'absorbe complètement sans possibilité pour elle de s'en distancer. Ces deux préoccupations relatives au contrôle d'un élève et à l'anticipation de la suite de la leçon confinent en quelque sorte le professeur stagiaire à l'immédiateté de la situation présente. L'arrivée des élèves munis de leurs tapis est un élément saillant pour elle, associant le caractère désordonné de la répartition des élèves et le sentiment d'être débordée. A cet instant, elle s'aperçoit qu'elle n'a pas énoncé les limites de répartition dans l'espace et les indique aux premiers élèves qui arrivent avec leurs tapis. Elle découvre dans cette situation que les autres élèves, dispersés, n'entendent pas ses indications.

Les réorganisations décrites au gré des diverses situations montrent que si Caroline semble avoir à sa disposition des moyens efficaces pour parvenir à regrouper les élèves les évènements la dépassent. Si elle semble également avoir déplacé sa perspective sur les raisons de ce flottement [les élèves ne sont pas autonomes (leçon1)/dire qu'il ne faut pas laisser d'espace libre n'est pas concret (entretien de formation post-leçon/leçon2)]. Soulignons que dans cette transition vers un nouvel équilibre son inconfort est encore plus important. Le fait d'avoir à sa disposition les moyens de parvenir à ses fins semble décupler ce sentiment.

\section{CAS n`3 - Caroline}

Au cours d'un exercice de gymnastique, Caroline donne des conseils à une élève qui la sollicite en affirmant qu'elle ne parvient pas à réaliser le mouvement demandé. Elle poursuit ensuite son déplacement à la rencontre d'autres élèves plus éloignés. Au cours de l'entretien, le formateur est surpris : "L'élève te dit : "Je ne sais pas faire ». Tu lui 


\section{eJRIEPS 34 janvier 2015}

donnes un conseil, mais tu ne restes pas... C'est surprenant ça... ». Le professeur stagiaire répond : « J'ai du mal à rester longtemps à regarder quelqu'un. J'ai toujours peur que ça dérape dans mon dos [que les élèves perturbent le déroulement de la leçon] ». Elle estime son action auprès des élèves efficace et la proposition du formateur étonnante : “ Je trouve surprenant qu'il me dise : "Tiens c'est surprenant que tu ne restes pas à côté d'elle » ». Elle interprète cette réaction en se disant que le formateur ne connaît pas assez ses élèves réputés difficiles à contrôler : "Si je reste à côté d'elle [l'élève en difficulté] ça va partir de tous les côtés, elle va mettre du temps à faire l'exercice et les autres vont en profiter ». Estimant ces élèves capables de perturber le déroulement de sa leçon, elle les surveille en permanence. Dans ces situations de classe, ses préoccupations sont de voir tous les élèves afin de leur donner des conseils, mais aussi d'être vue. Elle a appris, lors des leçons précédentes, que l'activité des élèves est d'autant plus importante qu'elle se trouve proche d'eux et qu'elle leur fait sentir sa présence par le regard. Elle a également repéré que les élèves se mettent en activité lorsqu'elle se dirige vers eux ou qu'elle leur adresse à distance de courtes injonctions.

A posteriori, cette interaction avec le formateur ne fait pas l'objet de réflexion. Le professeur stagiaire estime que sa façon d'intervenir lors de la leçon suivante est adéquate : «Je reste sur ce fonctionnement où je passe, je donne le conseil, je m'en vais... Ça ne me pose pas de problème dans ma pratique en fait... ». Elle récuse au cours de l'entretien la remarque insistante du formateur sans pour autant lui montrer de signes de désapprobation. Nous pouvons faire l'hypothèse qu'elle fuit le rapport de force avec le formateur dans la mesure où elle est convaincue de l'efficacité de sa façon d'intervenir en classe et a contrario de l'inefficacité de son conseil.

Dans le cas présent, le formateur semble ne pas avoir identifié la pluralité des préoccupations du professeur stagiaire pour conserver le contrôle des élèves et le maintien de leur travail en classe. Sa proposition de se centrer sur un élève entraînerait une focalisation sur une seule préoccupation ; ce que le professeur stagiaire refuse puisqu'elle ne contrôlerait plus l'ensemble des élèves. La description de la complexité de son activité en classe n'a pas lieu lors de l'entretien de formation (en présence du formateur) mais au cours de l'entretien d'autoconfrontation (en présence du chercheur). L'équilibre construit au fur et à mesure de ses expériences en classe lui donne satisfaction puisqu'il répond à ses fins. Et c'est sur cette différence de finalités de l'intervention en classe qu'achoppe le conseil du formateur. D'un côté, le professeur stagiaire tient à contrôler l'action des élèves et à les maintenir globalement au travail (ce qu'elle parvient à 


\section{eJRIEPS 34 janvier 2015}

faire) ; de l'autre, le formateur souhaite l'orienter vers un suivi plus individualisé des élèves (ce qu'elle n'envisage pas dans l'immédiat).

Le cas met en évidence les décalages entre les exigences des formateurs et les préoccupations des professeurs stagiaires et la difficile remise en question de l'activité lorsqu'elle est associée à un sentiment d'efficacité en classe. Les remarques répétées du formateur, appuyées sur le constat d'une efficacité limitée des professeurs stagiaires en termes d'apprentissage des élèves, sont sans effet sur leur activité.

\section{CAS n4 - Jérôme :}

Pour une première expérience d'enseignement de l'activité danse hip hop, Jérôme, enseignant expérimenté, a choisi un contexte sécurisant : une classe facile, un cycle de courte durée sur des séances d'une heure, nous sommes à la $5^{\text {ème }}$ séance d'un cycle de 8 ; sa préoccupation première est de « tenir » pendant tout le cycle sans perdre l'adhésion des élèves.

Dans la première partie de la leçon, il leur demande de reproduire un ensemble de mobilisations segmentaires et articulaires, puis de gestes codifiés empruntés aux techniques du hip hop ; il se place face aux élèves, en situation de modèle, démontre la gestuelle attendue et la réalise en même temps qu'eux. Le film de sa séance nous montre que les réponses des élèves ne correspondent pas au modèle proposé par l'enseignant. $\mathrm{Au}$ bout de quelques minutes de son autoconfrontation, Jérôme s'étonne de ne pas intervenir pour corriger les réponses qu'il juge inadéquates à la vidéo. Ainsi confronté aux traces de son activité, il découvre ce qu'il fait, mais aussi ce qu'il aurait pu faire et qu'il ne fait pas; il identifie que les choix réalisés ne sont pas les seuls possibles, ni même souhaitables et tente de trouver une signification à ses actes. II évoque un conflit de préoccupations : celle relative à l'anticipation sur le déroulement de son cours, prend le pas sur la préoccupation relative à l'observation et la régulation de l'action présente des élèves; il complète ensuite son interprétation en invoquant un manque de repères didactiques pour lire la motricité de ses élèves.

Lorsqu'il visionne la situation d'apprentissage suivante, conçue selon les mêmes modalités, il s'étonne de nouveau de ce qu'il appellera désormais sa « cécité didactique ". 


\section{eJRIEPS 34 janvier 2015}

Vignette $n^{\circ} 1$ : « on devient très compétent dans la cécité "

J/ La cécité didactique ! Mais ça prouve à quel point les préoccupations...déjà quand tu n'as pas de vécu dans l'activité, je pense que tu as énormément de mal à voir ce qui va pas chez les élèves ; comme ta préoccupation en plus est doublée par l'idée qu'il faut que ça tourne ... euh ...là on devient très compétent dans la cécité ; parce que j'ai 4 élèves qui sont à la rue : un, deux, trois, quatre...sur la vidéo, je pense un tout petit peu plus à l'arrivée, je le sais et en plus je le sais depuis le départ qu'ils sont à la rue, mais je ne mets rien en place pour ... [...] donc à un moment donné de ce point de vue là en tant que prof, je suis pas bon, j'étais peut être pas mauvais sur d'autres trucs mais là-dessus j'ai pas été bon; j'ai pas résolu leurs problèmes et je les ai laissés dans la panade...là je le vois bien là...

Ch : et là par rapport à ce que tu es en train de dire, ça veut dire que dans la séance tu le vois pas ou tu vois pas comment...

$\mathrm{J}$ : non non non non, je le vois pas, je le vois pas, je suis vraiment préoccupé par l'idée qu'il faut que ça tourne et que ce que j'ai prévu se fasse ; je pense pas, parce que quand on débute dans la carrière on peut se poser la question différemment, quand on a quand même 10 ou 11 ans de pratique, on a quand même a minima la capacité à proposer une situation qui va déverrouiller la chose [il énonce quelques pistes de régulation possibles] donc j'ai des billes mais j'ai une cécité là qui est pas liée au fait de pas avoir de billes pour intervenir sur la motricité c'est que j'ai , j'ai le souci de...que ça tourne et de pas me faire arrêter cette fois ci par 4 ou 5 élèves qui me bloquent. [...] c'est inconscient hein, je le verbalise parce qu'on le voit là, mais au moment où je l'ai fait...

$\mathrm{Ch} /$ oui c'est un compromis qui est à l'œuvre dans l'activité $\mathrm{J} /$ en fait voilà, on est en plein dans le compromis, je zappe les 4 élèves ...

Dans cet extrait, Jérôme se livre à une nouvelle interprétation de la situation : sa " cécité " n'est plus seulement liée à un déficit de ressources didactiques, elle s'apparente ici à une stratégie ("on devient très compétent dans la cécité ") ; le chercheur va alors le solliciter en lui demandant de préciser les raisons de l'empêchement de son activité en cours de séance. II permet ainsi à Jérôme d'aiguiser le regard qu'il porte sur son activité, et d'y trouver de nouvelles significations : cette " cécité » devient la traduction d'un compromis privilégiant la recherche d'une efficience dans l'usage de soi (" de pas me faire arrêter cette fois ci par 4 ou 5 élèves qui me bloquent») en acceptant, de "zapper quatre élèves » . 


\section{eJRIEPS 34 janvier 2015}

Un peu plus tard dans la même situation, il demande à un élève qu'il identifie en réussite de montrer l'exercice au reste de la classe, ce faisant, il n'intervient toujours pas directement auprès de ceux qui sont en échec.

Vignette 2 : «j'aurais peut être dû... »

$\mathrm{J}$ : C'est la continuité de la cécité ça, je vois ce qui va, j'ai envie de voir ceux qui vont bien et je ne veux pas voir ce qui ne vont pas, parce que ça...probablement, ça, ça, rentre dans ma logique de continuer la séance [...] par contre, j'ai pas forcément, euh, je me pose encore la question de savoir si il faut que systématiquement je le fasse avec eux ou que je prenne un peu plus de recul, ce que je fais dans d'autres séances et que je fais pas là...voilà [...] après se pose la question de savoir si finalement je suis, je m'obstine à vouloir systématiquement qu'ils m'aient comme repère, mais finalement, je pense que le fait d'avoir des repères plus loin comme je l'ai annoncé tout à l'heure, regarde devant, file devant, regarde devant, file devant, c'est plus intéressant que m'avoir moi comme repère où finalement, ils...(silence) ...ils s'approprient pas le geste par eux mêmes ; ils restent sur l'imitation, en fait et ça va peut être trop vite pour eux, même si on va tous au même rythme, va peut être trop vite pour eux, j'aurais peut être du...et puis moi, en tant que prof, j'aurais peut être été mieux derrière, pour voir plus de choses...

Ce nouvel extrait montre une évolution dans l'activité d'analyse de Jérôme : après un nouveau commentaire, cette fois sur le caractère sélectif de sa « cécité », il va remettre au travail sa préoccupation première et modalise ses interprétations relatives aux empêchements de son activité.

L'objet de son activité d'analyse s'est déplacé puisqu'il s'interroge désormais sur les instruments utilisés lors de cette séquence d'enseignement: la nature de la tâche prescrite et la forme de guidage utilisées, et sur leurs effets sur les apprentissages des élèves ; il se met à (re) penser ses occupations et à les envisager dans une perspective dynamique, c'est-à-dire « du point de vue des rapports mouvants entre ce qu'(il) fait, ce qu'(il) devrait faire, ce qu'(il) voudrait ou pourrait faire... » (Saujat, 2010, p102). Un tel renversement est susceptible d'ouvrir la voie à un développement potentiel du pouvoir d'agir, si l'on s'accorde avec Vygotski (1997) et sa métaphore du jeu d'échec sur l'idée qu'il y a développement lorsqu'un sujet perçoit les mêmes choses de son activité autrement, ouvrant ainsi d'autres possibilités d'action par rapport à elle. Devenu 


\section{eJRIEPS 34 janvier 2015}

observateur de sa propre activité, il va pouvoir réinterpréter la situation et mettre en perspective un renouvellement de ses occupations, autorisant ainsi un développement potentiel de ces dernières.

Ce cas met au jour la manière dont un enseignant d'EPS expérimenté, mais néophyte dans l'activité physique, sportive et artistique (APSA) qu'il doit enseigner, le hip hop, se trouve confronté à la résurgence de préoccupations typiques des enseignants débutants d'éducation physique, telles qu'elles ont été identifiées notamment par Ria (2001). Conserver le plan de la leçon, maintenir les élèves en action à travers des formes de travail traditionnelles, s'avèrent constituer des préoccupations qui pèsent fortement sur les choix de cet enseignant, créent des empêchements dans son activité, et sont à l'origine de discordances entre ce qui fait sens dans son activité d'enseignant d'EPS (guider les élèves dans leurs apprentissages moteurs pour leur permettre d'apprendre) et qui relève du genre professionnel de l'enseignant d'EPS expérimenté, et ce qu'il parvient à faire, qui se traduit par une efficacité relative en terme d'impact sur ses élèves et renvoie à un usage débutant de ce même genre.

Ce cas jette également une lumière intéressante sur le fait que, comme nous l'évoquions plus haut, le « genre débutants » ne concerne pas que les débutants, révélant la portée développementale que nous assignons à l'hypothèse d'un usage débutant du genre enseignant. En effet cette hypothèse ouvre plus largement sur l'étude des compensations mobilisées par des enseignants expérimentés, dont l'expertise est souvent présentée comme homogène, dès lors qu'ils sont confrontés à des transformations de leur travail, qu'elles proviennent de prescriptions «descendantes» (celles qui relèvent de l'organisation de ce travail) ou « remontantes" (celles qui viennent de l'objet même de leur travail). L'usage débutant du genre est ici mobilisé pour apprivoiser une APSA nouvelle avec les élèves, dont la mise en œuvre constitue une mise en danger pour l'enseignant. Cela amène des formes de protection qui sont liées à une activité prioritairement relative à soi-même et qui se traduisent par ce que cet enseignant qualifie au cours de l'autoconfrontation de "cécité didactique ». Le souci d'efficience et de confort professionnel prime celui de pertinence didactique des tâches proposées en hip hop, alors que c'est ce dernier qui prévaut lorsque ce professeur enseigne d'autres APSA. Le processus d'autoconfrontation, à travers les étonnements et les discordances vécues qu'il génère, permet de recycler les préoccupations initiales et d'imaginer d'autres possibilités d'action, orientées par d'autres intentions et réclamant d'autres opérations (Bertone et Saujat, 2013 ; Laurent et Saujat, à paraître). 


\section{eJRIEPS 34 janvier 2015}

\section{Discussion}

Les études de cas permettent d'ouvrir la discussion sur deux versants : les conditions du développement professionnel et les implications sur la formation. Si les possibilités et les empêchements de l'activité de l'enseignant ne sauraient être compris et/ou expliqués à partir des seules intentionnalités, quels repères se donner pour mieux comprendre les tensions entre déterminants et intentionnalités. Si les intentionnalités sont débordées par les épreuves subjectives à la source des compromis au moyen desquels l'enseignant s'efforce de donner corps à ses préoccupations à travers ses « occupations », quelles implications en tirer pour la formation ?

5.1. Élargir le champ des possibles : condition nécessaire mais pas suffisante Le développement professionnel est pour partie associé à l'idée d'un élargissement du champ des possibles. Or, l'élargissement du champ des possibles réside dans le rôle joué par ce que l'on pourrait appeler la double alternance dans les apprentissages et le développement professionnel des enseignants engagés dans ces contextes dialogiques d'analyse de leur activité (entretien post-leçon ou autoconfrontation). En effet, une alternance opère ici selon un double mouvement, externe et interne.

- « externe » : il est alors question d'alternance, relative au dispositif mis en place, entre expériences de classe et expériences liées aux processus d'interaction formative qui se déploient à la faveur du processus d'intervention (entretien de formation pour Caroline ou l'entretien d'auto-confrontation pour Jérôme).

- «interne »: liée cette fois à l'activité des protagonistes eux-mêmes, et plus particulièrement une alternance entre ce que l'enseignant fait dans son milieu ordinaire, la classe; ce qu'il dit dans le milieu extra-ordinaire d'entretien de formation (Caroline) ou d'autoconfrontation (Jérôme) de ce qu'il fait en classe et pour finir ce qu'il fait de ce qu'il dit lors de son retour en classe.

Les études de cas montrent de ce point de vue comment cette double alternance génère (a) des étonnements qui font « ré-agir » les protagonistes, (b) un déplacement et un recyclage des préoccupations et (c) des cycles récursifs d'apprentissage et de développement professionnels. Notons que l'élargissement des possibles est une condition nécessaire pour que les enseignants puissent « re-saisir » leur activité. Elle n'est pour autant pas suffisante pour que s'engage des cycles récursifs d'apprentissage et développement. Ce constat nous amène à envisager quelques implications pour la formation. 


\section{eJRIEPS 34 janvier 2015}

\subsection{Implications pour la formation}

L'étude des réorganisations de ces équilibres provisoires conduit à des implications pour la formation. Si se former "c'est accepter de modifier avec prudence le début de stabilité professionnelle à peine échafaudée" (Serres, 2006, p. 207), alors la formation doit permettre de recomposer avec les systèmes de normes qui pèsent sur l'activité à l'œuvre, produites par l'institution, par les formateurs, par le métier et par les individus eux-mêmes. L'activité des formateurs en prise directe avec les situations de travail est au cœur de cette tension entre intentionnalités et déterminants. Selon notre approche développementale de l'activité, envisagée dans son historicité et sa potentialité, leur intervention pourrait s'inscrire à plusieurs niveaux pour aider les enseignants : 1) à redéfinir les prescriptions depuis leurs formulations les plus générales à leurs déclinaisons les plus spécifiques, 2) à envisager la façon de les réaliser dans des scénarios pédagogiques envisagés en amont des situations d'enseignement, 3) à analyser rétrospectivement les tensions entre ce qu'on leur demande et ce que ça leur demande en vue d'envisager des déplacements de perspective, 4) à envisager des projections vers de nouveaux équilibres acceptables entre efficacité objective et subjective, 5) à accompagner les moments de transitions d'un équilibre à l'autre.

Dans cette perspective, le travail des formateurs réside notamment dans leur capacité à s'ajuster sans s'assujettir, c'est-à-dire à prendre en considération les intentionnalités des acteurs sans perdre celles de l'institution. Si un travail au plus proche du vécu semble nécessaire pour accéder aux « préoccupations ", il semble tout aussi nécessaire d'aider à leur reprise dans des "occupations" susceptibles de faire évoluer favorablement les enseignants mais aussi les situations de classe.

Sur un autre plan, l'attention portée aux intentionnalités semble aller de pair avec une valorisation des activités réflexives. S'il semble nécessaire dans l'intervention de susciter des questions, il semble tout aussi nécessaire de susciter des réponses aux questions suscitées de façon à instruire les questions de métiers. Pour le dire autrement, si élargir le champ des possibles est une condition nécessaire au développement, elle n'est pas suffisante. Si l'entretien de formation ou l'auto-confrontation dans les études de cas proposées ont provoqué des déplacements, une ouverture sur de nouveaux possibles, il ne s'agit là que de potentialités portées par des individus isolés. A quelles conditions ces questionnements suscités chez tel ou tel individu peuvent-ils être instruits et portés par le métier? 


\section{eJRIEPS 34 janvier 2015}

5.3 Quels repères pour l'intervention entre déterminants et intentionnalités ? Une des limites de l'accompagnement professionnel des enseignants tient à nos yeux à une conception « visualiste » du développement centrée sur les gestes professionnels des enseignants sans les mettre systématiquement en relation avec la part moins visible des déterminants et des intentionnalités. Nos études de cas permettent de pointer le caractère discret de certaines évolutions dans l'activité: évolution des intentionnalités, « déplacements » de perspectives, évolution des significations. Travailler sur les tensions entre déterminants et intentionnalités est une voie prometteuse à nos yeux dans une perspective de formation. Une des difficultés des formateurs de terrain qui œuvrent au plus proche des pratiques de classe est d'intervenir sur la base d'une observation de pratique avec un accès très limité aux intentionnalités et au rapport qu'elles entretiennent avec les tensions vitales de l'activité. Nos observations et nos analyses nous amènent donc à soutenir les interventions formatives susceptibles de prendre conjointement en charge les intentionnalités et les déterminants de l'activité d'enseignement.

\section{Références}

Amigues, R., Faïta, D. \& Saujat, F. (2004). Travail enseignant et organisation de l'apprentissage scolaire. In E. Gentaz et P. Dessus (Eds.). Comprendre les apprentissages. Sciences cognitives et éducation (pp. 155-168). Paris : Dunod.

Amigues, R., Félix, C. \& Saujat, F. (2008). Les connaissances sur les situations d'enseignement-apprentissage à l'épreuve des prescriptions ? Les Dossiers des Sciences de l'Education, 19, 27-39.

Bakhtine, M. (1984). Esthétique de la création verbale. Paris : Gallimard.

Bertone, S. et Saujat, F. (2013). La réflexivité comme instrument de formation par alternance et de développement de l'activité professionnelle des enseignants. Activités, 10(2), 177-192, http://www.activites.org/v10n2/v10n2.pdf

Bruner, J. (1983). Le développement de l'enfant : savoir faire, savoir dire. Paris : PUF.

Canguilhem, G. (1966/1983). Le concept et la vie. In G. Canguilhem, Etudes d'histoire et de philosophie des sciences (pp. 72-87). Paris : Vrin.

Clot, Y. (1999/2002). De Vygotski à Leontiev, via Bakhtine. In Y. Clot (Ed.). Avec Vygotski. Paris : La Dispute.

Clot, Y. (2011). Théorie en clinique de l'activité. In B. Maggi (Ed.). Interpréter l'agir : un défi théorique (pp. 17-39). Paris : PUF. 


\section{eJRIEPS 34 janvier 2015}

Daguzon, M. \& Goigoux, R. (2007). L'influence de la prescription adressée aux professeurs des écoles en formation initiale : construction d'un idéal pédagogique. CDRom Actes du colloque AREF : Strasbourg. http://www.congresintaref.org/actes site.php

Daniellou, F. (2002). Le travail des prescriptions. Conférence inaugurale au 37ème Congrès de la SELF, les évolutions de la prescription, Aix-en-Provence. http://www.ergonomie-self.org/actes/congres2002.html

Darré, J.P. (1994). Le mouvement des normes, avec Bakhtine et quelques agriculteurs. In J.P. Darré (Ed.). Pairs et experts dans l'agriculture. Dialogues et production de connaissances pour l'action. Toulouse : Erès.

Durand, M. (1996). L'enseignement en milieu scolaire. Paris: PUF.

Durand, M. (2009). La conception d'environnements de formation sous le postulat de l'enaction. In Durand, M. \& Filliettaz, L. (2009). Travail et formation des adultes (pp191-215). Paris : PUF

Goigoux, R. (2007). Un modèle d'analyse de l'activité des enseignants. Education et Didactique, 3, 1, 19-41.

Maggi, B. (2003). De l'agir organisationnel. Un point de vue sur le travail, le bien-être, l'apprentissage. Toulouse : Octarès.

Mayen, P. Savoyant, A. (2002) Formation et prescription: une réflexion de didactique professionnelle. $37^{\text {ème }}$ Congrès de la SELF, les évolutions de la prescription, Aixen-Provence 25,26 et 27 Septembre. Actes en lignes http://www.ergonomieself.org/actes/congres2002.html

Nonnon, E. (2000).Le tableau noir de l'enseignant, entre écrit et oral. Repères, 22, 83-119.

Ria, L. (2001). Les préoccupations des enseignants débutants en Education Physique et Sportive. Thèse de Doctorat, Université de Montpellier I.

Ria, L. (2004). Expériences typiques des enseignants débutants. EPS, 305, 67-70.

Ria, L. (2009). De l'analyse de l'activité des enseignants en milieu difficile à la conception de dispositifs de formation. In M. Durand et L. Filliettaz (Eds.). Travail et formation des adultes (pp. 217-243). Paris : PUF.

Roustan, C. \& Saujat, F. (2008). «Genre débutant » et co-construction d'un milieu pour l'étude: le cas du football au cours moyen (9-10 ans). Travail \& Formation en Education, 1/2008. Mis en ligne le 2 décembre 2008. URL: http://tfe.revues.org/index616.html. 


\section{eJRIEPS 34 janvier 2015}

Rubinstein, S. L. (2007). Nouvelles figures de l'activité humaine. Anthologie de textes choisis et édités par V. Nosulenko et P. Rabardel. Toulouse : Octarès-Editions Maison des sciences de l'homme.

Saujat, F. (2004). Comment les enseignants débutants entrent dans le métier. Formation et pratiques d'enseignement en questions. Revue des HEP de Suisse Romande et du Tessin, 1, 97-106.

Saujat, F. (2008). Le métier d'enseignant. In S. Chaliès et S. Bertone (Eds.). L'enseignement (pp. 45-61). Paris : Editions Revue EPS

Saujat, F. (2010). Travail, formation et développement des professionnels de l'éducation : voies de recherche en sciences de l'éducation. Note de synthèse pour l'Habilitation à Diriger des Recherches. Université de Provence - Aix-Marseille I

Saujat, F. (2011). L'activité enseignante. In B. Maggi (Ed.). Interpréter l'agir : un défi théorique (pp. 241-257). Paris : PUF.

Saujat, F., Faïta, D. \& Maggi, B. (2006). Travail et santé des enseignants : synergie entre deux méthodes pour comprendre un objet méconnu et transformer les situations d'enseignement. Actes du 41e Congrès de la SELF : Ergonomie et Santé au travail (pp. 163-167). www.ergonomie-self.org/self/listcong.html

Schwartz, Y. (1992). Travail et philosophie. Convocations mutuelles. Toulouse : Octarès.

Schwartz, Y. (2000). Le paradigme ergologique ou un métier de philosophe. Toulouse: Octarès.

Serres, G., Ria, L. et Adé, D. (2004). Modalités de développement de l'activité professionnelle au gré des contextes de classe et de formation : le cas des professeurs stagiaires en Education Physique et Sportive. Revue Française de Pédagogie, 149, 49-64.

Serres, G. (2006). Analyse de la construction de l'expérience professionnelle au gré des diverses situations de formation initiale des enseignants du second degré. Thèse de Doctorat en Sciences de l'Education. Clermont-Ferrand: Université Blaise Pascal.

Six, F. (1999). De la prescription à la préparation du travail : apports de l'ergonomie à la prévention et à l'organisation du travail sur les chantiers de bâtiment. Note pour l'Habilitation à Diriger des Recherches. Lille : Université Charles de Gaulle Lille 3.

Tardif, M. \& Lessard, C. (1999). Le travail enseignant au quotidien. Expérience, interactions humaines et dilemmes professionnels. De Boeck Université. 
eJRIEPS 34 janvier 2015

Vérillon, P. (2005). Les artefacts matériels et sémiotiques comme organisateurs de l'activité. Document de travail (4 pages).

Vygotski, L. (1994). Défectologie et déficience mentale. Lausanne : Delachaux et Niestlé.

Vygotski, L. (1997). Pensée et langage. Paris : Editions Sociales. 